\title{
Strong binding of Leupeptin with TMPRSS2 protease may be an alternate to Camostat and Nafamostat for SARS-CoV-2 repurposed drug: Proof from Molecular docking and Molecular dynamics simulations
}

\author{
Jaganathan Ramakrishnan $^{1}$, Saravanan Kandasamy ${ }^{1}$, Ancy Iruthayaraj ${ }^{1}$, SIVANANDAM \\ MAGUDEESWARAN $^{1}$, Kalaiarasi Chinnasamy ${ }^{1}$, and Kumaradhas Poomani ${ }^{1}$
}

\author{
${ }^{1}$ Periyar University
}

May 15, 2020

\begin{abstract}
The unprecedented coronavirus SARS-CoV-2 outbreak at Wuhan, China caused acute respiratory infection to humans. There is no precise vaccine/therapeutic agents available to combat the COVID-19 disease. Some repurposed drugs are saving the life of diseased, but the complete cure is relatively less. Several drug targets have been reported to inhibit the SARS-CoV-2 virus infection, in that TMPRSS2 (Transmembrane protease serine 2) is one of the potential targets, inhibiting this protease stops the virus entry into the host human cell. Camostat mesylate, nafamostat and leupeptin are the drugs, in which the first two drugs are being used for COVID-19 and leupeptin also tested. To consider these as repurposed drugs for COVID19 , it is essential to understand their binding affinity and stability with TMPRSS2. In the present study, we performed the molecular docking and molecular dynamics (MD) simulation for the three molecules with the TMPRSS2. The docking study reveals that leupeptin molecule strongly binds with TMPRSS2 protein than the other two drug molecules. The RMSD and RMSF values of MD simulations shows, leupeptin and the amino acids of TMPRSS2 are very stable than the other molecules. Furthermore, leupeptin forms interactions with the key amino acids of TMPRSS2 and the same have been maintained during the MD simulations. This structural and dynamical information is useful to evaluate these drugs to be used as repurposed drugs, however, the strong binding profile of leupeptin with TMPRSS2, suggests, it may be considered as a repurposed drug for COVID-19 after clinical trial.
\end{abstract}

\section{Introduction}

For the past three decades, the outbreak of new viruses causing strange epidemic diseases and high mortality. Among these viruses, notably, Influenza, Hepatitis, and HIV viruses are high replicative in nature and no drugs available to stop the infection and give a complete cure. Coronavirus is a RNA virus which was emerged and evolved to different types of coronaviruses. ${ }^{[1,2]}$ Among these, the outbreak of the SARS-CoV virus in 2002 made a major health threat to the public and caused Severe Acute Respiratory Syndrome (SARS), it resulted in 774 deaths over the 8098 infected cases. ${ }^{[3,4]}$ The evolution of coronavirus was continuous, in the year 2012, another version of Middle East Respiratory Syndrome-CoV (MERS-CoV) virus also emerged, which is found to be vulnerable and the outbreak of the MERS-CoV virus made high health emergency in the Middle East countries, infected 2506 people and resulted in 862 deaths. ${ }^{[5,6]}$ Further, recently, a new type of coronavirus also again emerged in Wuhan, China in December, 2019, which was identified as a novel coronavirus SARS-CoV-2 namely COVID-19, which causes severe acute respiratory infection (pneumonia) and other health problems. ${ }^{[7-9]}$ As on date, nearly 4 million people were infected, in which 2,84,034 people died. Such high mortality rate may be due to several reasons, however, it is widely accepted that the mode 
of infection of SARS-CoV-2 is different from the SARS-CoV and MERS-CoV. As per reports SARS-CoV-2 is a beta coronavirus, its genome sequence is $79 \%$ identical to SARS-CoV and $50 \%$ identical to MERS-CoV. ${ }^{[10]}$ SARS-CoV-2 is an enveloped single positive-stranded RNA virus. The viral structure consists of structural proteins such as spike $(\mathrm{S})$, membrane $(\mathrm{M})$, envelope (E) and nucleocapsid $(\mathrm{N})$. The spike S-protein presents in the envelope of the virus mediates the viral particle into the host-cells. M-protein is largely present in the virion and jointly with E-protein forms a mature viral envelope. N-protein always binds with RNA, which is required for the packing of viral RNA. ${ }^{[11,12]}$ SARS-CoV-2 initiates human cell entry after the spike (S) protein present on the envelope binds to a cell membrane receptor called the angiotensin converting enzyme (ACE2). The S-protein is cleaved into two subunits S1 and S2, by a human cell-derived protease (thought to be Furin), subsequently, S1 binds to its receptor, ACE2. The other fragment, S2, is cleaved by TMPRSS2 (Transmembrane protease serine 2), a human cell surface serine protease, resulting in membrane fusion. Both ACE2 and TMPRSS2 are therefore thought to be essential in airway cells for SARS-CoV-2 infection. Inhibiting TMPRSS2 protein using suitable inhibitors can stop the virus entry and this prevents the SARS-CoV-2 virus infection. The present study is focused to find the inhibitors of TMPRSS2 protein from the existing drugs. ${ }^{[13-17]}$ Camostat mesylate, nafamostat, and leupeptin (Figure 1) are the drugs chosen as the inhibitors of TMPRSS2. Among these, camostat and nafamostat are already been in clinical trials and using for COVID-19 ${ }^{[13,16,18-21]}$, and Leupeptin ${ }^{[22]}$ also tested for SARS-CoV-2 virus infection. To understand the molecular mechanism of binding of these molecules is not completely known at molecular dynamics level; hence, we have computed the binding affinity, intermolecular interactions, and stability of molecules from the molecular docking and the molecular dynamics simulations. The information obtained from this computational study is useful to consider these drugs as a repurposed drug for the therapeutic agents of COVID-19, however it is subjected to clinical studies and other requirements.

\section{Materials and Methods}

Homology modeling and Molecular docking

The crystal structure of TMPRSS2 is not available, hence the homology model structure was created from the amino acid sequence of TMPRSS2 with catalytic domain was retrieved from the UniprotKB (Uniprot Accession O15393) using Schrodinger homology modeling interface ${ }^{[23]}$; in which, Hepsin structure (PDB: $5 \mathrm{CE} 1$ ) was used as a template, based on the sequence similarity (BLAST). ${ }^{[24]}$ Finally, the modeled structure was optimized and refined by protein preparation wizard. ${ }^{[25]}$. The structure was validated with the Ramachandran plot (Figure 1S). ${ }^{26]}$

To understand the binding affinity and the intermolecular interaction of chosen three drug molecules camostat, nafamostat and Leupeptin ${ }^{[16,18-21]}$ with the active site amino acids of the TMPRSS2, the molecular docking has been performed. In prior to the docking simulation, the three drug molecules were prepared by the Ligprep module of Schrodinger software ${ }^{[27]}$ Further, these molecules were docked with the TMPRSS2 by extra precision mode (XP) incorporated in the induced fit docking (IFD) method. The IFD resulted in different conformations for each ligand-TMPRSS2 complex. Based on the top score with the lowest energy conformation and intermolecular interactions, the ligand-TMPRSS2 complex was selected for each case and used for further study. PyMOLsoftware ${ }^{[28]}$ was used to analyze the intermolecular interactions between ligand and protein for the individual complex.

Molecular dynamics simulation and binding free energy

To understand the stability, intermolecular interactions, and the binding energy of the three ligand-TMPRSS2 complexes, the molecular dynamics (MD) simulation has been performed using the OPLS3e force field [29] implemented in Desmond v5package ${ }^{[30]}$. Further, the system was built with the pre-defined TIP4P water model and orthorhombic periodic boundary conditions at the distances $10 \AA$. Then the counterions were used to neutralize the charge of these complexes with the balancing $\mathrm{Na}^{+} / \mathrm{Cl}^{-}$ions. Further, the constructed system for each ligand-TMPRSS2 complex was energy minimized by heating and equilibrium processes before the MD simulation. For the minimization, the minimization and heating protocol was fixed based on the steepest descent method, annealing temperature at 0-300 K, and 2000 steps with the time steps of 0.001 
ps. Further, the system was normalized in an equilibrium state at 1000 steps with a time step of 0.001 ps. Finally, the production step of the systems was continued up to $100 \mathrm{~ns}$ with the time steps of $0.001 \mathrm{ps}$, $300 \mathrm{~K}, 1$ ATM pressure, and applied using the Nose-Hoover method with NPT ensemble ${ }^{[31]}$. Intermolecular interactions and conformation of each ligand-protein complex was analyzed from the final results of MD simulation. Among the 1000 fractions, 10, 20, 30, 40, 50, 60, 70, 80, 90, and 100 ns fractions were used to determine the binding free energy (MM/GBSA) of the ligand-TMPRSS2 complexes by Primeapplication incorporated to Schrodinger software package ${ }^{[32]}$.

\section{Results and Discussion}

Molecular docking simulation and Ligand(s) -TMPRSS2 binding

TMPRSS2 is the serine protease, which mediates the S-protein of SARS-CoV-2 for the virus entry through the ACE2 enzyme. Reportedly, by inhibiting the TMPRSS2 protease, the SARS-CoV-2 fusion can be stopped $^{[13-17]}$. In TMPRSS2, the ligand-binding active site has catalytic triad consists of Ser186, His41, and Asp180 amino acids, to inhibit the function of this enzyme, ligands should mainly bind with these key amino acids. The molecular docking simulations for the drug molecules casmostat, nafamostat and leupeptin with TMPRSS2 have been performed using induced fit docking (IFD) method, the docking score values of the three molecules are $-6.648,-7.075$ and $-9.325 \mathrm{kcal} / \mathrm{mol}$ respectively, whereas the IFD scores are -490.01 , -493.20 and $-501.23 \mathrm{kcal} / \mathrm{mol}$. And the glide energy values $-57.457,-56.96$ and $-64.35 \mathrm{kcal} / \mathrm{mol}$ (Table 1). On compare the above-said values reveal that, consistently, leupeptin exhibits high values. The magnitude of difference in docking scores and glide energy reflects the nature of their intermolecular interactions with the neighboring amino acids present in the active site of TMPRSS2 protease (Figure 2). In the catalytic site, the camostat and leupeptin are forming strong interactions with Asp180, Ser186 and His41 than the nafamostat(Table 2); however, the interactions of leupeptin with the catalytic amino acids Asp180, Ser186 and His41are found stronger and the interaction distances are 1.9, 2.1, 2.0 and 3.5 A respectively.Figure 3 shows the superimposed form of the three drug molecules in the active site of TMPRSS2, reveal the orientation of the molecules and the difference. From this, we observed that the three molecules have a tendency to bind with the serine 2 protease TMPRSS2. However, the nafamostat is lacking the Ser186 and His41 interactions with TMPRSS2 (Table 2). Further, we have probed the existence of these interactions, stability, and the binding affinity of the three drug molecules with the TMPRSS2 during the molecular dynamics simulations.

\section{Stability of ligand(s)-TMPRSS2 complexes and Intermolecular interactions}

Molecular dynamics is crucial to consider for the understanding of the biological function of proteins. Here we performed $100 \mathrm{~ns}$ MD simulations for the three ligand-TMPRSS2 complexes to explore the stability and the binding affinity of the three drug molecules towards the TMPRSS2.Figure $4 \& \mathbf{5}$ displays the variation of RMSD and RMSF values of the three complexes during the MD simulations. The RMSD values of the three drug molecules casmostat, nafamstat, and leupeptin are remains within 2 A (Figure 4 (A-C), whereas the RMSD of protein in the camostat-protein and nafamostat-protein complexes are found high on compare with leupeptin bound protein complex (2.4 A). Figure 5 (A-C) shows the variations of RMSF of amino acids TMPRSS2 of three complexes; among these, a strong fluctuation is found in the region of residue number 60 of camostat -TMPRSS2 complex and no such incidence is noticed in the RMSF map of other two complexes. The superimposed form of the three ligand-protein complexes (Figure 6) obtained from the docking and MD simulations is showing the conformational difference of ligands and TMPRSS2 protein in the respective complex; specifically, this allows to visualize that how the conformation and the orientation of the ligands and proteins are altered during the MD simulation. Overall, the fluctuation of the leupeptin molecule is found to be very less and it is normal. Furthermore, surprisingly, the superimposed form of docked and MD complexes of leupeptin (Figure 6) is showing not much deviation as the other two complexes deviates considerably; this confirms that leupeptin is very stable while binds with TMPRSS2 protein.

The intermolecular interactions between the ligand and the active site amino acids of TMPRSS2 of three 
complexes obtained from the MD simulations are shown in (Figure 7 (A-C)). On compare these interactions with the interactions found in the docked ligand-TMPRSS2 complexes (Figure 2), shows that, during the MD simulation some interactions became strong and few new interactions also formed(Figure 7). Further, when we examine the interactions present in the camostat-TMPRSS2 complex, the terminal $\mathrm{NH}_{2}$ group of camostat molecule forms interactions with one of the key amino acids Asp180 at the distances 1.6 and $1.7 \mathrm{~A}$ and these interactions are also very stable during the MD simulation. Whereas in the nafamostat-TMPRSS2 complex, we observed a similar trend, in which the molecule forms interactions with Asp180 at the distances 2.0 and $1.9 \mathrm{~A}$; in addition that it also forms an interaction with His41, the contact distance is $2.3 \mathrm{~A}$. And these two molecules also form several interactions with the nearby amino acids present in the active site. In the leupeptin-TMPRSS2 complex, as found in the docked complex, the MD simulation also reproduced the hydrogen bonding interactions between leupeptin and the key amino acids Asp180, His41 and Ser186 at the distances, 1.8, 1.8 and $1.7 \mathrm{~A}$. These important interactions were highly stable throughout the 100 ns MD simulations and this can be well understood from theFigures 8(A-C), 9(A-C) \& Figure 2S. The characterization of interactions between the drug molecules and TMPRSS2, reveals that the leupeptin molecule is highly stable and forms the expected interactions with the key amino acids Asp180, His41, and Ser186 of catalytic site and are found very strong. Whereas the other two drug molecules camostat and nafamostat are lacking the interactions with His41 and Ser186 amino acids. The binding energy for the three complexes were calculated using MM-GBSA method. The calculated values of the binding energy of camostat, nafamostat, and leupeptin molecules with TMPRESS2 are $-42.77,-52.86$, and $-55.25 \mathrm{kcal} / \mathrm{mol}$. Among these molecules, relatively, leupeptin has a high binding affinity with the TMPRSS2 serine protease. Overall leupeptin exhibits high stability and forms very strong interactions with the key amino acids than the other two drug molecules.

\section{Conclusions}

To meet out the urgent need of drugs to treat the COVID-19 pandemic disease, repurposing drugs is the only feasible solution. In the line of drug searching process, we have undertaken camostat, nafamostat and leupeptin drug molecules to inhibit the TMPRSS2 serine 2 protease, which is the activator of SARS-CoV-2 for the fusion of the virus enter the host human cell. The molecular docking study reveals that these three drug molecules form interactions and has binding affinity towards TMPRSS2. However, the leupeptin forms strong interactions with the key amino acids Ser186, His41, and Asp180 of catalytic triad present in the active site of TMPRSS2 as the other two molecules lacking Ser186 and His41 interactions. Whereas in the molecular dynamics simulations of these three complexes, leupeptin is highly stable (RMSD \& RMSF) and this molecule also forms stable interactions with the key amino acids Ser186, His41, and Asp186 of TMPRSS2 during the $100 \mathrm{~ns}$ MD simulations. On compare the binding free energy of all the three molecules, notably, leupeptin has high binding affinity towards TMPRSS2. From the static and dynamic studies, it is confirmed that leupeptin is very stable and it could strongly inhibit the TRMPRSS2 serine 2 protease and this leads to stop the fusion of SARS-CoV-2 virus into the host human cell, hence it may be consider as a repurposed drug after clinical studies.

\section{Acknowledgements}

The authors thank the Computer Centre, Periyar University, Salem to perform the computational work in the High Performance Cluster (HPC) Computer.

\section{References}

1. Woo PC, Huang Y, Lau SK, Yuen K.Y. Coronavirus Genomics and Bioinformatics Analysis. Viruses . 2010;2:1804-1820.

2. Woo PC, Lau SK, Lam CS, Lau CC, Tsang AK, Lau JH, Bai R, Teng JL, Tsang CC, Wang M, Zheng BJ, Chan KH, Yuen KY. Discovery of seven novel Mammalian and avian coronaviruses in the genus deltacoronavirus supports bat coronaviruses as the gene source of alphacoronavirus and betacoronavirus and avian coronaviruses as the gene source of gammacoronavirus and deltacoronavirus. $J$ Virol . 
2012;86:3995-4008.

3. Kuiken T, Fouchier RA, Schutten M, Rimmelzwaan GF, van Amerongen G, van Riel D, Laman JD, de Jong T, van Doornum G, Lim W, Ling AE, Chan PK, Tam JS, Zambon MC, Gopal R, Drosten C, van der Werf S, Escriou N, Manuguerra JC, Stohr K, Peiris JS, Osterhaus AD. Newly discovered coronavirus as the primary cause of severe acute respiratory syndrome.Lancet . 2003;362:263-270.

4. Zhong NS, Zheng BJ, Li YM, Poon, Xie ZH, Chan KH, Li PH, Tan SY, Chang Q, Xie JP, Liu XQ, Xu J, Li DX, Yuen KY, Peiris, Guan Y. Epidemiology and cause of severe acute respiratory syndrome (SARS) in Guangdong, People's Republic of China, in February, 2003. Lancet.2003;362:1353-1358.

5. Zumla A, Hui DS, Perlman S. Middle East respiratory syndrome.Lancet . 2015; 386:995-1007.

6. Aleanizy FS, Mohmed N, Alqahtani FY, El Hadi Mohamed RA. Outbreak of Middle East respiratory syndrome coronavirus in Saudi Arabia: a retrospective study. BMC Infect Dis . 2017;17, 23.

7. Wang C, Horby PW, Hayden FG, Gao GF. A novel coronavirus outbreak of global health concern. Lancet . 2020;395:470-473.

8. Huang C, Wang Y, Li X, Ren L, Zhao J, Hu Y, Zhang L, Fan G, Xu J, Gu X, Cheng Z, Yu T, Xia J, Wei Y, Wu W, Xie X, Yin W, Li H, Liu M, Xiao Y, Gao H, Guo L, Xie J, Wang G, Jiang R, Gao Z, Jin Q, Wang J, Cao B. Clinical features of patients infected with 2019 novel coronavirus in Wuhan, China. Lancet. 2020;395: 497-506.

9. Zhou P, Yang XL, Wang XG, Hu B, Zhang L, Zhang W, Si HR, Zhu Y, Li B, Huang CL, Chen HD, Chen J, Luo Y, Guo H, Jiang RD, Liu MQ, Chen Y, Shen XR, Wang X, Zheng XS, Zhao K, Chen QJ, Deng F, Liu LL, Yan B, Zhan FX, Wang YY, Xiao GF, Shi ZL. A pneumonia outbreak associated with a new coronavirus of probable bat origin. Nature. 2020;579:270-273.

10. Lu R, Zhao X, Li J, Niu P, Yang B, Wu H, Wang W, Song H, Huang B, Zhu N, Bi Y, Ma X, Zhan F, Wang L, Hu T, Zhou H, Hu Z, Zhou W, Zhao L, Chen J, Meng Y, Wang J, Lin Y, Yuan J, Xie Z, Ma J, Liu WJ, Wang D, Xu W, Holmes EC, Gao GF, Wu G, Chen W, Shi W, Tan W. Genomic characterisation and epidemiology of 2019 novel coronavirus: implications for virus origins and receptor binding. Lancet. 2020;395:565-74.

11. Neuman BW, Adair BD, Yoshioka C, Quispe JD, Orca G, Kuhn P, Milligan RA, Yeager M, Buchmeier MJ. Supramolecular Architecture of Severe Acute Respiratory Syndrome Coronavirus Revealed by Electron Cryomicroscopy. J Virol. 2006; 80:7918-7928.

12. Barcena M, Oostergetel GT, Bartelink W, Faas FG, Verkleij A, Rottier PJ, Koster AJ, Bosch BJ. Cryo-electron tomography of mouse hepatitis virus: Insights into the structure of the coronavirion. PNAS. 2009;106:582-587.

13. Hoffmann M, Kleine-Weber H, Kruger N, Muller M, Drosten C, Pohlmann S. The novel coronavirus 2019 (COVID-19) uses the SARS-1 coronavirus receptor ACE2 and the cellular protease TMPRSS2 for entry into target cells. BioRxiv. (2020)Preprintdoi: https://doi.org/10.1101/2020.01.31.929042

14. Glowacka I, Bertram S, Muller MA, Allen P, Soilleux E, Pfefferle S, Steffen I, Tsegaye TS, He Y, Gnirss K, Niemeyer D, Schneider H, Drosten C, Pohlmann S. Evidence that TMPRSS2 activates the severe acute respiratory syndrome coronavirus Spike protein for membrane fusion and reduces viral control by the humoral immune response. J Virol. 2011;85:4122-4134.

15. Iwata-Yoshikawa N, Okamura T, Shimizu Y, Hasegawa H, Takeda M, Nagata N (2019) TMPRSS2 contributes to virus spread and immunopathology in the airways of murine models after coronavirus infection. J Virol 2019;93(6):pii: e01815-18.

16. Kawase M, Shirato K, van der Hoek L, Taguchi F, Matsuyama S. Simultaneous treatment of human bronchial epithelial cells with serine and cysteine protease inhibitors prevents severe acute respiratory syndrome coronavirus entry. J Virol. 2012; 86:6537-6654.

17. Zhou Y, Vedantham P, Lu K, Agudelo J, Carrion R Jr, Nunneley JW, Barnard D, Pohlmann S, McKerrow JH, Renslo AR, Simmons G. Protease inhibitors targeting coronavirus and filovirus entry. Antiviral Res . 2015;116:76-84.

18. https://clinicaltrials.gov/ct2/show/NCT04353284

19. Bittmann S, Luchter E, Weissenstein A, Villalon G, Moschuring-Alieva E. TMPRSS2-Inhibitors Play a role in Cell Entry Mechanism of COVID-19: An Insight into Camostat and Nafamostat. Regen Biol 
Med . 2020;2(2):1-3.

20. Hoffmann M, Schroeder S, Kleine-Weber H, Muller MA, Drosten C and Pohlmann S. Nafamostat mesylate blocks activation of SARS-CoV-2: New treatment option for COVID-19. Antimicrob. Agents Chemother . 2020 (In press). DOI: 10.1128/AAC.00754-20

21. Sonawane KD, Barale SS, Dhanavade MJ, Waghmare SR, Nadaf NH, Kamble SA, Mohammed AA, Makandar AM, Fandilolu PM, Dound AS, Naik NM. Homology modeling and docking studies of TMPRSS2 with experimentally known inhibitors Camostat mesylate, Nafamostat and Bromhexine hydrochloride to control SARS-Coronavirus-2.ChemRxiv .(2020)Preprint. https://doi:org/10.26434/chemrxiv.12162360.v1

22. Appleyard G, Tisdale M. Inhibition of the Growth of Human Coronavirus 229E by Leupeptin. J Gen Virol. 1985;66:363-366.

23. Schrodinger Release 2020-1: Prime, Schrodinger, LLC, New York, NY, 2020.

24. Altschul SF, Gish W, Miller W, Myers EW, Lipman DJ. Basic local alignment search tool. J Mol Biol . 1990;215:403-410.

25. Schrodinger Suite 2012 Protein Preparation Wizard; Epik version 2.3, Schrodinger, LLC, New York, NY, 2012; Impact version 5.8, Schrodinger, LLC, New York, NY, 2012; Prime version 3.1, Schrodinger, LLC, New York, NY, 2012.

26. Ramachandran GN, Ramakrishnan C, Sasisekharan V. Stereochemistry of polypeptide chain configurations. J Mol Biol. 1963;7:95-9.

27. Schrodinger Release 2020-1: LigPrep, Schrodinger, LLC, New York, NY, 2020.

28. The PyMOL Molecular Graphics System, (2002). Version 2.0 Schrodinger, LLC.

29. Harder E, Damm W, Maple J, Wu C, Reboul M, Xiang JY, Wang L, Lupyan D, Dahlgren MK, Knight JL, Kaus JW, Cerutti D, Krilov G, Jorgensen WL, Abel R, Friesner RA. OPLS3: a force field providing broad coverage of drug-like small molecules and proteins. J Chem Theory Comput.2016;12:281-296.

30. Desmond Molecular Dynamics System, D. E. Shaw Research, New York, NY, 2020. Maestro-Desmond Interoperability Tools, Schrodinger, New York, NY, 2020.

31. Evans DJ, Holian BL. The Nose-Hoover thermostat. J Chem Phys.1985;83:4069.

32. Li J, Abel R, Zhu K, Cao Y, Zhao S, Friesner RA. The VSGB 2.0 model: a next generation energy model for high resolution protein structure modelling.Proteins. 2011;79:4-812.
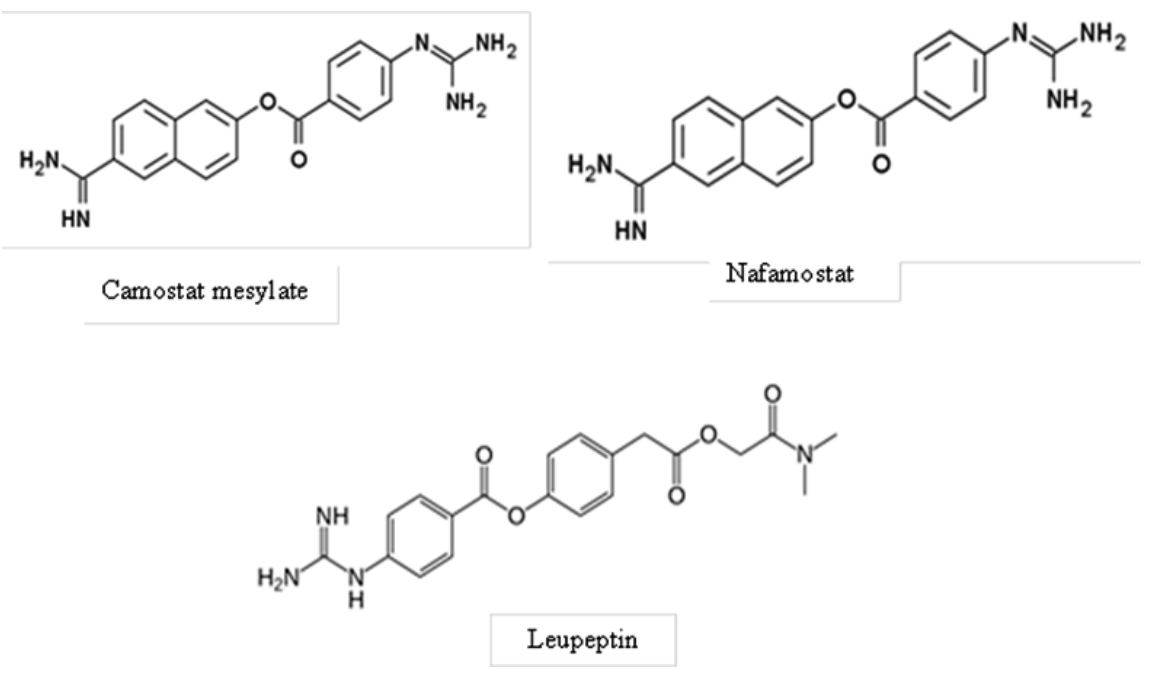

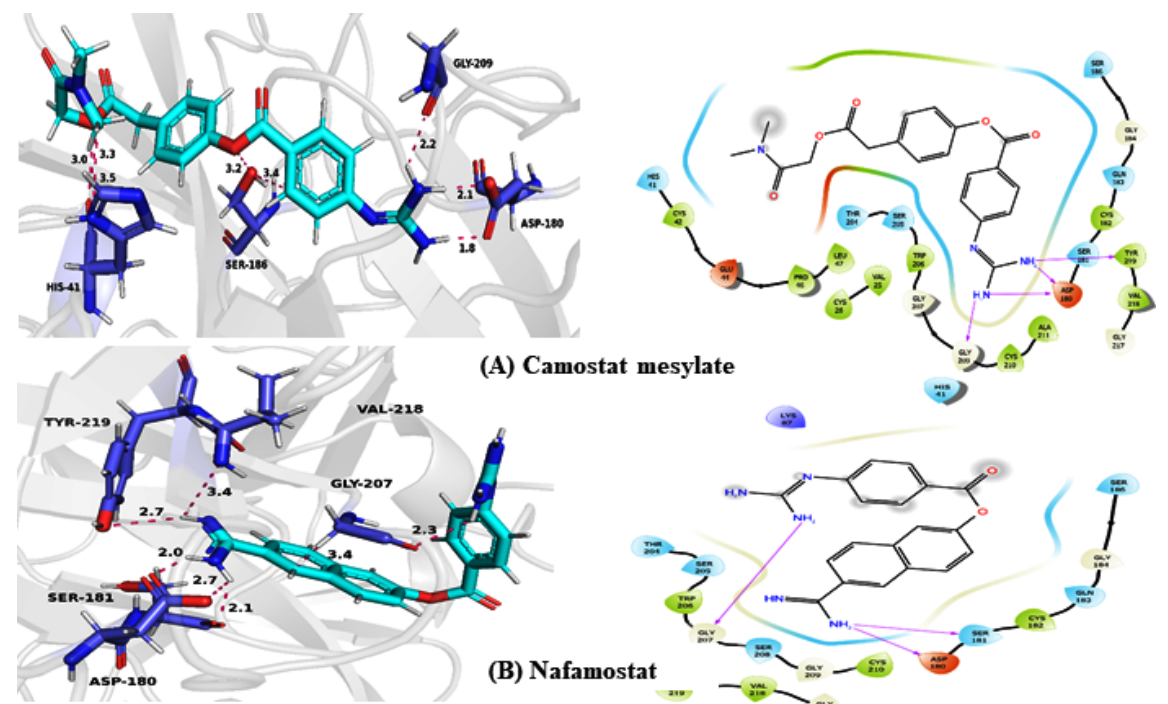

Camostat mesylate
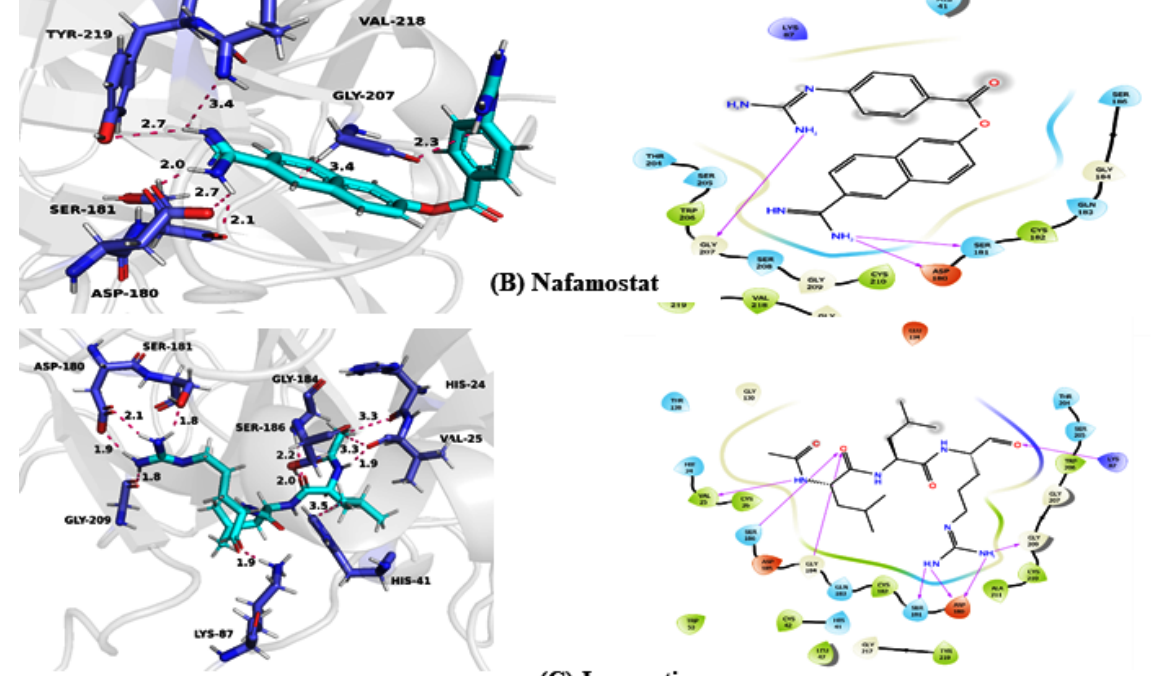

B) Nafamosta

(C) Leupeptin
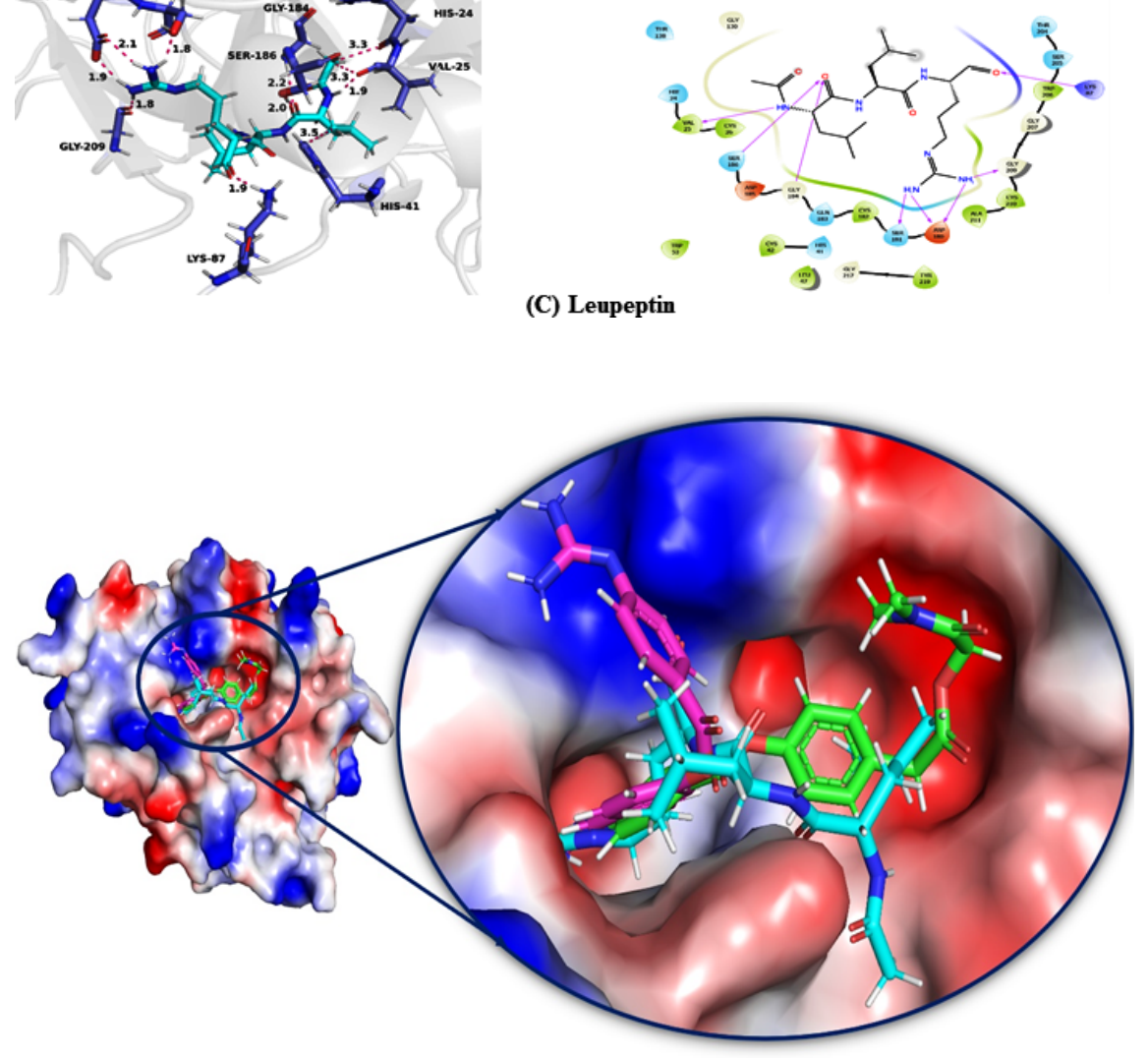

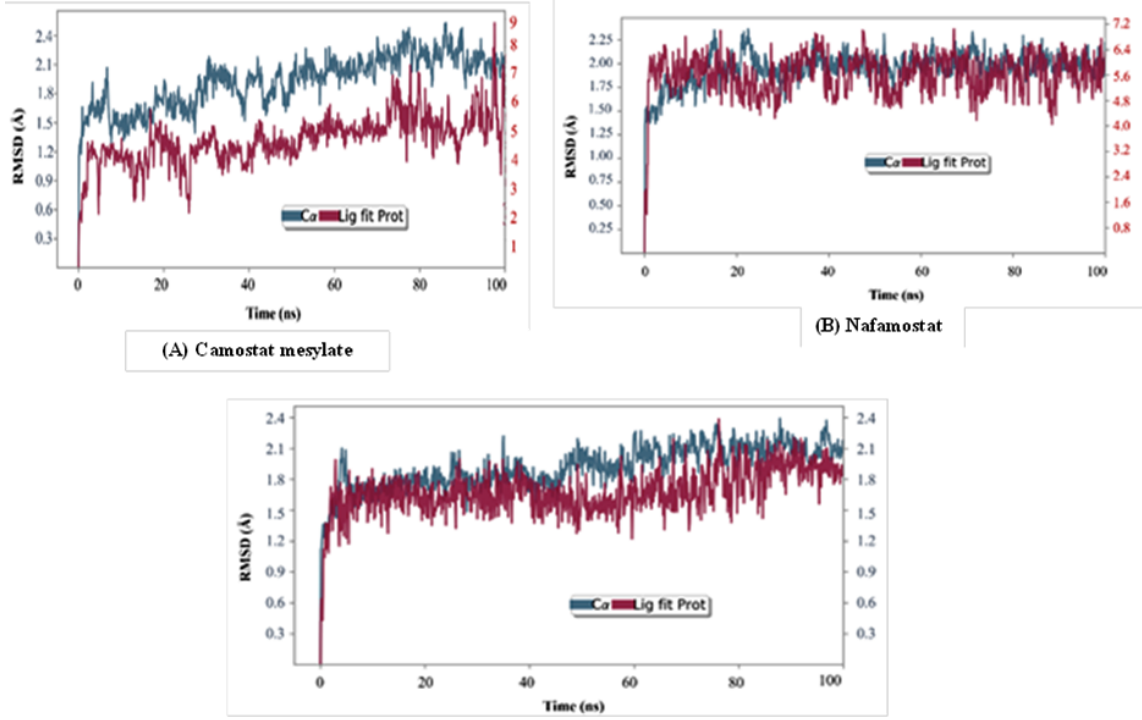

(C) Leupeptin
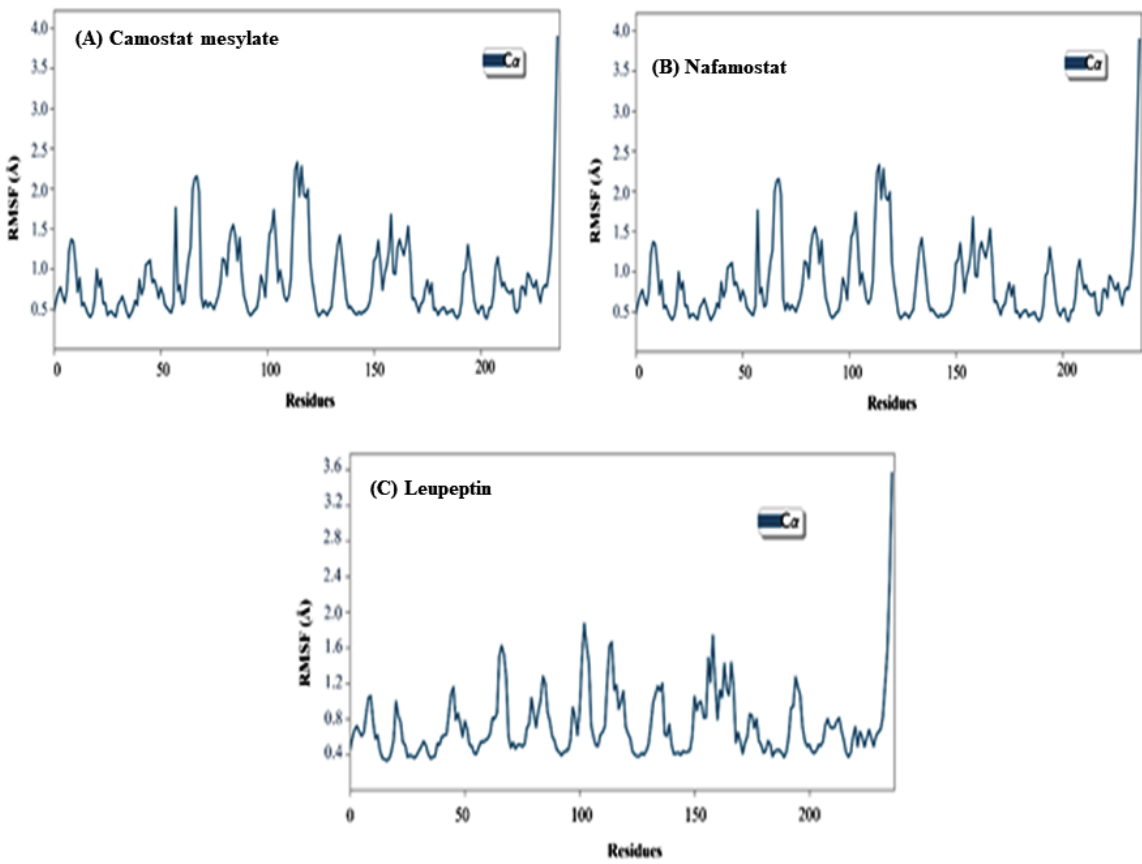

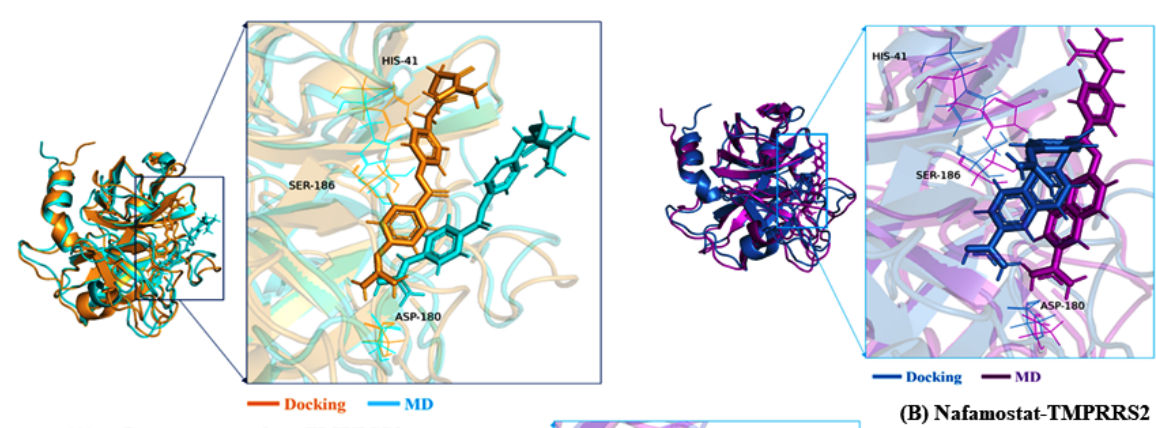

(A) Camostat mesylate-TMPRSS2

(B) Nafamostat-TMPRRS2

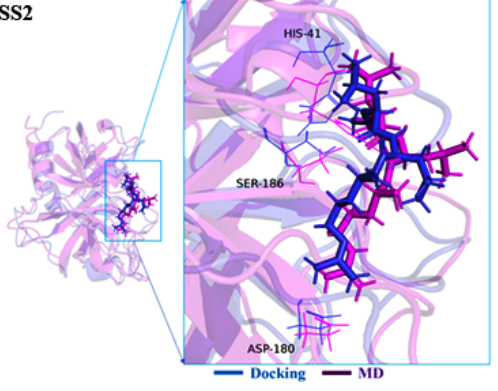

(C) Leupeptin-TMPRSS2
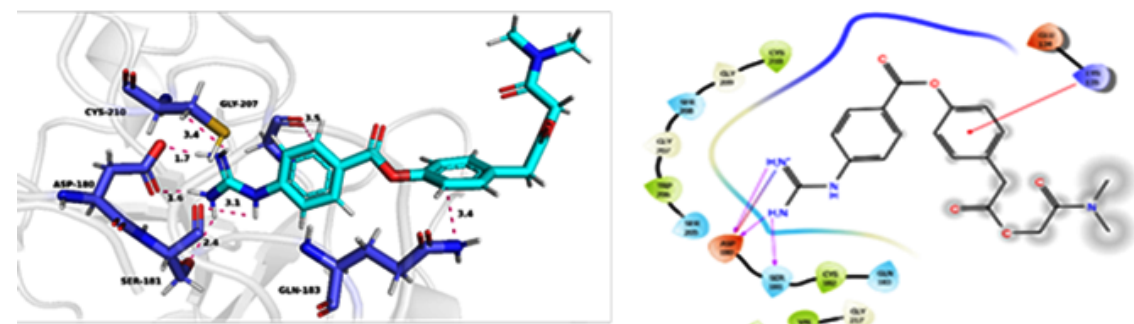

(A) Camostat mesylate
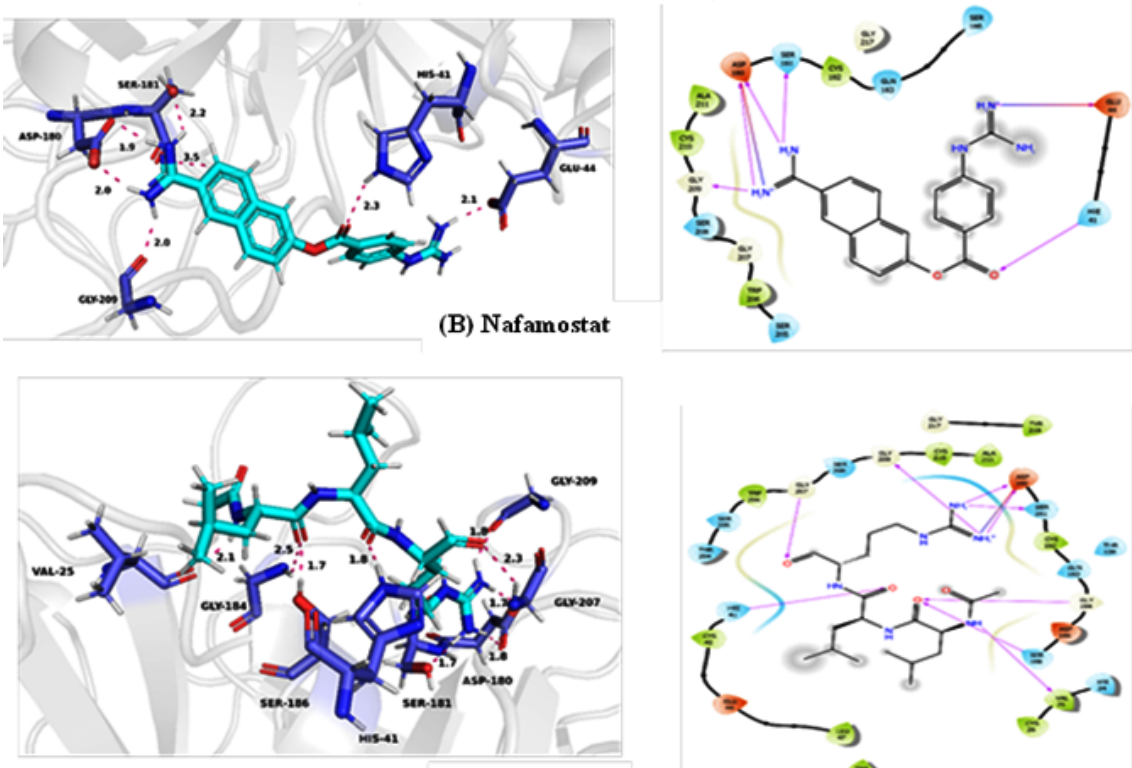

(C) L eup eptin

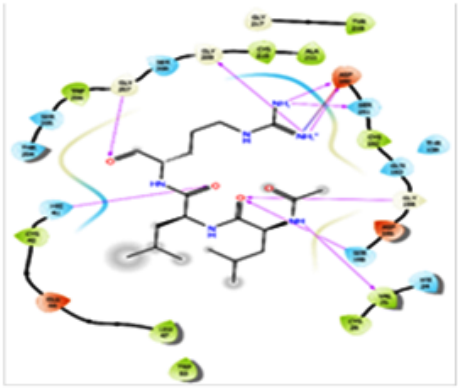




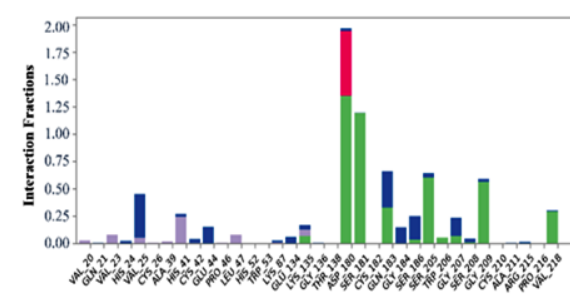

$\square$ H-bonds $\square$ Hydrophobic $\square$ lonic $\square$ Water bridges

(A) Camostat mesylate

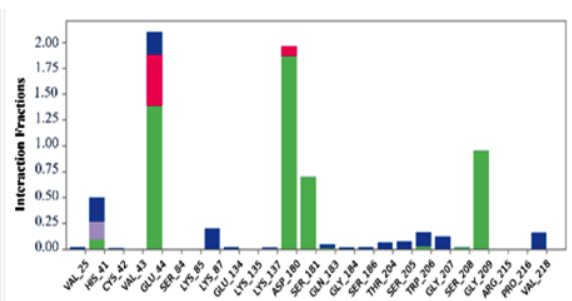

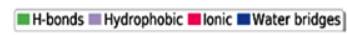

(B) Nafamostat

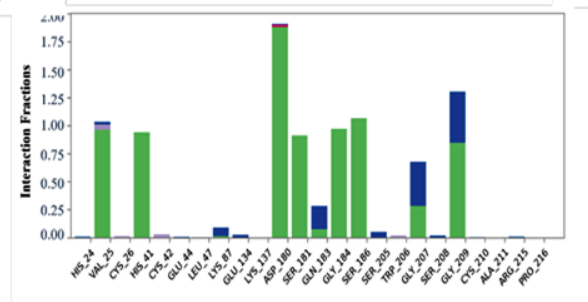

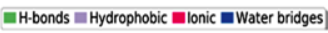

(C) Leupeptin

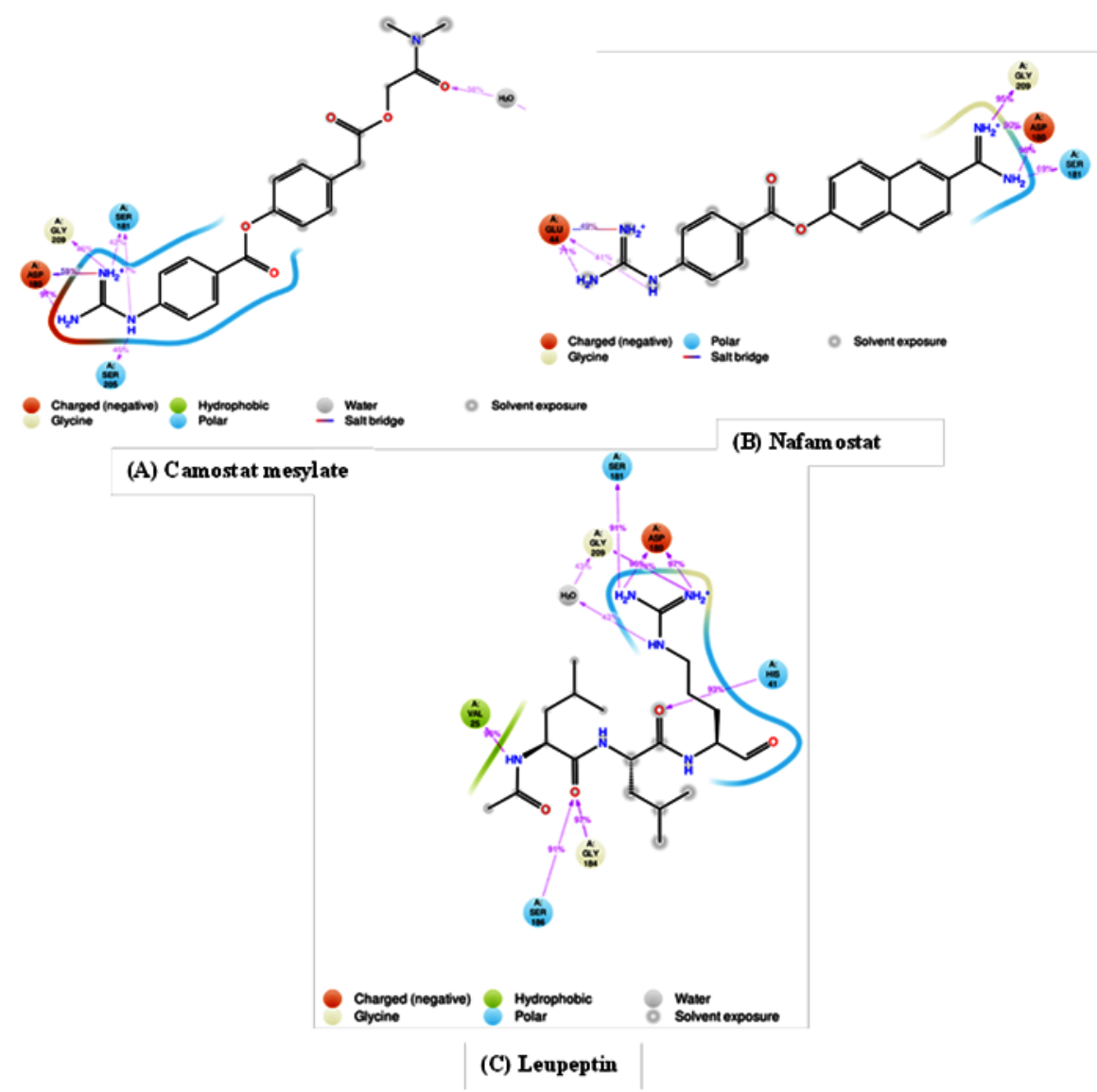

Hosted file

Tables.doc available at https://authorea.com/users/322417/articles/451388-strong-binding-of- 
leupeptin-with-tmprss2-protease-may-be-an-alternate-to-camostat-and-nafamostat-for-sarscov-2-repurposed-drug-proof-from-molecular-docking-and-molecular-dynamics-simulations 